\title{
6 \\ Plague Today
}

\section{ELISABETH CARNIEL}

This chapter contains a brief overview of plague today (current foci, epidemiology, clinical symptoms, causative agent, treatment, and prophylaxis), as seen from the viewpoint of the medical and scientific community. It is not an "argument" but a description of our current observations and understanding of the disease.

Three plague pandemics are traditionally described during the Christian era. The first pandemic, designated "the plague of Justinian", broke out in Egypt in 541; the second, known as the "Black Death", erupted in Messina, Sicily, in 1347; and the third began in the war-torn Yunnan province of China and reached Hong Kong in 1894. The network of steamships and railways favoured a rapid spread of the disease over all continents and the colonization of previously unscathed areas. Major advances in the knowledge of plague were made during the early years of the last pandemic. In 1894, Alexandre Yersin identified the aetiologic agent (Yersinia pestis) and showed that rats are a reservoir of the disease. ${ }^{1}$ Four years later, Paul-Louis Simond demonstrated that plague is transmitted by fleas. ${ }^{2}$ Thanks to the advent of effective public health measures, mass vaccination in 1934, and antibiotic therapy in 1938, the number of victims reported during the third pandemic was in no way comparable to those of the previous two.

\section{Plague Today}

Despite a considerable reduction in mortality and morbidity, plague has not been eradicated. Endemic foci persist in Africa, North and South America, and Asia. During the nineteen years from 1985 to 2003, over 43,000 human cases were reported to the World Health Organization by twenty-five countries. ${ }^{3}$ The worldwide numbers are most likely underestimated because of unrecognized cases and the failure of some countries to report occurrences of plague.

Since the beginning of the 1990s, a sharp rise in the number of human plague cases has been observed (Figure 1). This rise is partly attributable to the reappearance of epidemics in countries or regions where human plague had disappeared for several decades. This was the case in 1991 in the seaport of Mahajanga (Madagascar) after an absence of over sixty years, in 1994 in Malawi, Zimbabwe, Mozambique and India (where no case had been reported during nearly thirty years), in 1996 in Zambia and some provinces of China, in 1997 in Jordan (with no plague cases during eighty years), and more recently in Algeria (2003)

Elisabeth Carniel, MD, PhD, Institut Pasteur, Yersinia Research Unit, 28 rue du Dr Roux, Paris, France.

\footnotetext{
${ }^{1}$ Alexandre Yersin, 'La peste bubonique à Hong-Kong', Ann. Inst. Pasteur, 1894, 8: 662-7.

${ }^{2}$ Paul-Louis Simond, 'La propagation de la peste', Ann. Inst. Pasteur, 1898, 12: 625-87.

${ }^{3}$ WHO, 'Human plague in 1998 and 1999', Wkly. Epidemiol. Rec., 2000, 42: 337-44; WHO, 'Human plague in 2002 and 2003', Wkly. Epidemiol. Rec. 2004, 79: 301-8.
} 


\section{Elisabeth Carniel}

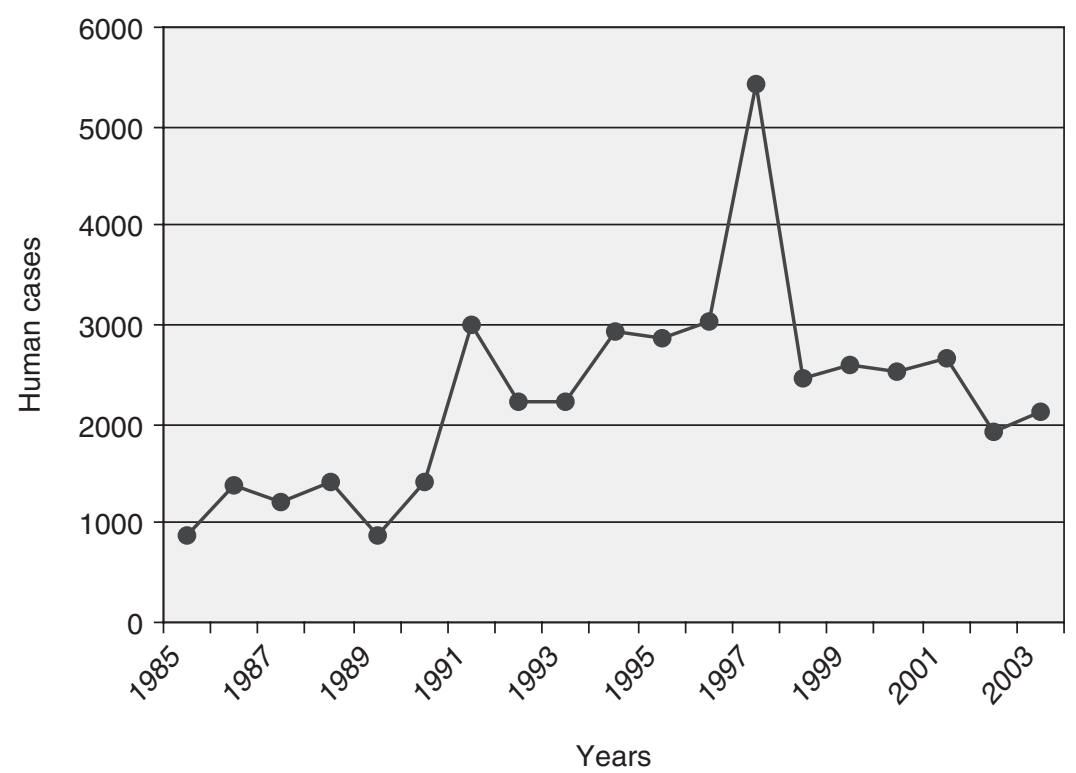

Figure 1: Annual numbers of human plague cases reported to WHO between 1985 and 2003.

after over fifty years with no outbreaks. For this reason, the plague is now categorized as a reemerging disease. ${ }^{4}$

\section{Plague: Epidemiology}

\section{Reservoir}

Plague primarily affects rodents but several other animal species including cats, rabbits, camels and humans can also be infected. Over 200 rodent species in 73 genera worldwide are susceptible in various degrees to the disease. Sporadically or periodically, explosive outbreaks occur among populations of susceptible rodents. The animal species constituting the natural plague reservoir vary greatly depending on the geographical location of the foci. For instance, rats represent the common source of human plague cases in Madagascar and Vietnam, while large gerbils in central Kazakhstan and meriones in Iran are the main reservoirs.

\section{Vector}

Plague is generally transmitted via the bites of fleas. Maintenance of plague in nature is totally dependent upon the cyclical transmission between fleas and mammals. More than eighty different species of flea are implicated in maintaining the plague cycle. These species and their transmission efficacy vary from country to country, depending on the characteristics of the ecological niches (reservoir species, climate, etc.).

\footnotetext{
${ }^{4}$ Stephanie J Schrag and Pamela Wiener, 'Emerging infectious diseases: what are the relative roles of ecology and evolution?', Trends Evol. Ecol., 1995, 10 (8): 319-24.
} 


\section{Plague Today}

While feeding on an infected host at a bacteremic/septicaemic stage, the ectoparasite ingests the bacilli present in the bloodstream. The bacteria multiply in the mid gut of the insect, eventually forming a solid mass that blocks its proventriculus. ${ }^{5}$ The flea that most commonly becomes blocked is the rat flea, Xenopsylla cheopis. During repeated attempts to feed on a new host, the hungry blocked flea is unable to pump blood into its stomach and subsequently regurgitates the bacteria into the bite wound.

The accepted paradigm over the last several decades was that only blocked fleas could efficiently transmit the disease. However, recent work has demonstrated that Oropsylla montana, the flea commonly found on highly plague-susceptible ground and rock squirrels, and the primary vector to humans in North America, can efficiently and rapidly transmit the disease without any blockage of its proventriculus. ${ }^{6}$ These results also suggest that the human flea, Pulex irritans, very prevalent on individuals during the Middle Ages, but which was disregarded as a potential human-to-human vector because of its poor blocking capacity, may actually have played a key role in the rapid spread of the Black Death.

\section{Foci}

Plague foci exist in a number of countries with landscapes, climates and altitudes that vary considerably. The central Kazakhstan focus, for instance, is a large zone of dry desert at sea level with very hot summers and extremely cold winters. This is completely different to the Madagascar focus, which is at high altitude $(>1000 \mathrm{~m})$ and has a temperate climate with sometimes high humidity and often a rich vegetation. ${ }^{7}$ Numerous intermediate landscapes between these two extremes exist in the world. These various ecological niches determine the different types of reservoirs and flea vectors, and consequently influence the incidence of human plague.

In almost all plague foci, animal and human infections are not found all the year round but predominate during the so-called "plague season". This season is usually constant in a given area, but differs from focus to focus, depending on the environmental characteristics (climate, altitude, etc.). ${ }^{8}$ Variations in seasonality can even be observed within the same country. In Madagascar for instance, the plague season is between October and March on the high plateaux, and between July and November on the coast. ${ }^{9}$ Similarly, a difference in seasonality is observed for the coastal and high plateau foci of Vietnam.

\section{Human Infections}

Rodent-to-human plague transmission most commonly occurs via infected fleas associated with peridomestic animals (rats, cats) or wild rodents. Although rare, infection may

\footnotetext{
${ }^{5}$ A W Bacot and C J Martin, 'Observations on the mechanisms of the transmission of plague by fleas', J. Hygiene, 1914, 13 (suppl. 3): 423-39.

${ }^{6}$ R J Eisen, S W Bearden, A P Wilder, J A Montenieri, MF Antolin, and K L Gage, 'Early-phase transmission of Yersinia pestis by unblocked fleas as a mechanism explaining rapidly spreading plague epizootics', Proc. Natl. Acad. Sci. USA, 2006, 103 (42): 15380-85.

${ }^{7}$ Suzanne Chanteau (ed.), Atlas de la peste à Madagascar, Paris, IRD, Institut Pasteur, AUF, 2006.

${ }^{8}$ Robert Pollitzer, Plague, Geneva, World Health Organization, 1954, pp. 486-90.

${ }^{9}$ Chanteau (ed.), op. cit., note 7 above, p. 53.
} 


\section{Elisabeth Carniel}

also result from direct exposure (through conjunctiva or cutaneous excoriations) to contaminated fluids or by inhalation of infected aerosols, for instance during the manipulation of animal furs. Human-to-human transmission may occur by inhalation of infected respiratory droplets spread by a patient suffering from pneumonic plague.

Differences in the age and sex incidence of bubonic plague cases are observed but are due merely to differences in the degree of exposure of the various groups to the infection rather than to intrinsic causes. In India, females were more frequently infected, whereas in Manchuria males were at higher risk of infection. ${ }^{10}$ Within the same country, the sex ratio may differ between urban and rural areas. Similarly, although plague may more commonly affect one age group, this group is not the same everywhere. Usually, adolescents and adults are considered the most at risk, but the infection predominates in children in several foci such as those of Madagascar and Brazil.

\section{Plague: Clinical Symptoms}

\section{General Symptoms}

The two major clinical forms of plague are bubonic and pneumonic. ${ }^{11}$ In both forms, the incubation period usually varies from a few hours to five or six days, although longer times (up to ten or fifteen days) have sometimes been reported. In the majority of plague attacks, the onset of the disease is sudden and severe. It is characterized by a rapid rise in temperature, which reaches $39.5^{\circ} \mathrm{C}$ to $40^{\circ} \mathrm{C}$ in a few hours, accompanied by an incessant quickening of the pulse and a fall in blood pressure, indicative of progressive heart failure. Symptoms of alteration of the nervous system are very common and show individual variations characterized by delirium and restless agitation in some patients, and apathy and stupor in others. Vomiting is considered a usual symptom by some physicians, but not so by others. Unless adequate specific treatment is administered, the condition of the patient deteriorates rapidly and death most commonly occurs within three to five days.

\section{Bubonic Plague}

Bubonic plague, the most common form of the disease, is acquired after the bite of an infected flea. At the site of the bite, the bacteria multiply and sometimes cause a small vesicle that develops into a painful, dark and necrotic carbuncle. Although not always present, this skin lesion is a valuable indication for the clinical diagnosis of plague. The bacilli then disseminate via the lymphatic vessels and reach the proximal draining lymph node where they multiply, causing an extremely tender tumefaction known as a bubo. Although the bubo usually forms at one site only, bilateral and multiple locations are also observed. In the best cases, the bubo spontaneously suppurates and the patient recovers from the infection. Most frequently, the bacterium disseminates via the blood vessels to the spleen, liver and sometimes the lungs, causing septicaemia that is soon fatal. In patients suffering from a severe type of the infection, localized or diffused skin haemorrhages

\footnotetext{
${ }^{10}$ Pollitzer, op. cit., note 8 above, pp. 503-4.

${ }^{11}$ Ibid., pp. 409-82.
} 


\section{Plague Today}

(petechiae or ecchymoses) may be a frequent complication. In the absence of appropriate treatment, the mortality rate of bubonic plague victims ranges from 40 to 70 per cent.

\section{Pneumonic Plague}

Although less frequent, pneumonic plague is an even more severe form of the disease. Individuals contract it through inhalation of infected droplets spread by a patient who had developed a lung infection, either as a secondary complication of a bubonic form, or after a primary pulmonary contamination. In addition to the general symptoms described above, the patients exhibit signs of lung involvement such as cough, muco-purulent and bloody sputum, dyspnoea and pain in the chest. Without treatment, the outcome of pneumonic plague is invariably fatal, usually in less than three days. However, in a number of instances, survival for nine to ten days has been recorded.

\section{Other Clinical Forms of Plague}

Besides the classic symptoms characteristic of bubonic and pneumonic plague, Yersinia pestis infection may show various clinical presentations in which septicaemic, meningeal, haemorrhagic, gastro-intestinal, or pharyngeal symptoms predominate. Benign, fulminant and even chronic forms of plague may also exist. Despite this large array of potential clinical symptoms, the plague is, nonetheless, a disease which is easily recognized, not only by physicians, but also by health agents and the population living in endemic areas.

\section{Plague: The Aetiological Agent}

The agent of plague, Yersinia pestis, is a small Gram-negative bacillus belonging to the genus Yersinia. This genus is part of the family Enterobacteriaceae and comprises twelve species, of which only three are human pathogens: Yersinia pestis, Yersinia pseudotuberculosis and Yersinia enterocolitica. Yersinia pseudotuberculosis and Yersinia enterocolitica differ epidemiologically and clinically from Yersinia pestis as they are enteropathogenic bacteria transmitted by the oral route. Despite strikingly different clinical manifestations and epidemiological cycles, DNA-DNA hybridization experiments indicated that Yersinia pestis is genetically almost identical to Yersinia pseudotuberculosis ( $>90$ per cent chromosomal relatedness). ${ }^{12}$ Recently, multilocus sequence typing confirmed the high genetic relatedness between Yersinia pestis and Yersinia pseudotuberculosis and showed that Yersinia pestis is a highly clonal species. ${ }^{13}$

In species where horizontal genetic exchange is rare, sequence polymorphism reflects the accumulation of mutations at a uniform clock rate and correlates with the time elapsed since the existence of a last common ancestor. Using the combination of two different

\footnotetext{
${ }^{12}$ Hervé Bercovier, et al., 'Intra- and interspecies relatedness of Yersinia pestis by DNA hybridization and its relationship to Yersinia pseudotuberculosis', Current Microbiology,1980, 4: 225-9.

${ }^{13}$ M Achtman, K Zurth, G Morelli, G Torrea, A Guiyoule, and E Carniel, 'Yersinia pestis, the cause of plague, is a recently emerged clone of Yersinia pseudotuberculosis', Proc. Natl. Acad. Sci. USA, 1999, 96 (24): 14043-48.
} 


\section{Elisabeth Carniel}

clock rates, it was possible to establish that the Yersinia ancestor appeared 42 to 187 million years ago and that Yersinia enterocolitica and Yersinia pseudotuberculosis diverged from their common ancestor 0.4 to 1.9 million years ago. Most importantly, this study showed that Yersinia pestis is a very recent clone, which emerged from Yersinia pseudotuberculosis within the last 1500 to 20,000 years.

\section{Plague: Diagnosis}

Bacteriological identification of Yersinia pestis is carried out on biological samples such as bubo aspirates, blood, sputum, cerebrospinal fluids or organs (liver, spleen, lungs) following post-mortem examination. Rapid presumption of plague infection can be obtained by Wayson staining or by a fluorescent antibody test, but confirmation of the diagnosis requires the isolation of the organism. This bacteriological identification may take up to one week and cannot be performed by health agents in the field.

Other techniques have been developed over the last few years to improve the rapidity of plague diagnosis. One of the most commonly available techniques is based on DNA detection by polymerase chain reactions (PCR). However, the sensitivity of this technique directly applied on biological samples is not always satisfactory and PCR is not applicable in most field laboratories. A rapid diagnostic test (15 minutes) has recently been developed and validated in the field. This dipstick does not require any sophisticated equipment and is now used routinely by public health agents in Madagascar. ${ }^{14}$

Serological techniques may be useful for retrospective diagnosis when a treatment has been administered to the patient without biological specimens being taken or when no plague bacilli have been isolated from these specimens. Today, the most widely used serodiagnostic test is based on enzyme-linked immunosorbent assay (ELISA).

\section{Plague: Treatment and Prevention}

\section{Vaccines}

The first mass vaccination campaigns against plague were carried out simultaneously in 1934 by Georges Girard and Jean-Marie Robic (strain EV) in Madagascar and by L Otten (strain Tjiwidej) in Java with live attenuated plague bacilli. ${ }^{15}$ These campaigns resulted in a significant decrease in human morbidity and mortality, but the adverse reactions and the short-term protection conferred by these vaccines led to a gradual decline in their utilization.

Formalin-killed plague organisms have also been used to induce immunity in humans, but the relatively low degree and short duration of protection, along with the adverse effects conferred by these vaccines, considerably limited their use. Today, several acellular or cellular recombinant vaccines are under study but they have not yet been tested in the natural conditions of endemic and epidemic plague foci.

\footnotetext{
${ }^{14}$ S Chanteau, L Rahalison, L Ralafiarisoa, J Foulon, M Ratsitorahina, L Ratsifasoamanana, E Carniel, F Nato, 'Development and testing of a rapid diagnostic test for bubonic and pneumonic plague', Lancet, 2003, 361: 211-16.

${ }^{15} \mathrm{G}$ Girard and J-M Robic, 'La vaccination de l'homme contre la peste au moyen de bacilles vivants (virus vaccin EV) son application à Madagascar', Bull. Off. Int. Hyg. Publ. 1936: 28, 1078-87; L Otten, 'Immunization against plague with live vaccine', Indian J. Med. Res., 1936, 24: 73-101.
} 


\section{Plague Today \\ Prophylactic Therapy}

Prophylactic treatments are administered to close contacts of pneumonic plague patients or to relatives of bubonic plague patients living in the same house. The treatment consists most often in the administration of sulphonamides (single or repeated doses) or tetracycline.

\section{Antibiotic Therapy}

The chances of patients' survival are directly linked to the speed with which antibiotic therapy is given, especially in the more severe pneumonic and septicaemic forms of the disease. The use of antibiotics to treat plague, which started in 1938 with sulphonamide and in 1946 with streptomycin, led to a dramatic drop in human mortality. With the current utilization of effective antibiotic therapy, the worldwide fatality rate attributable to plague has fallen down to approximately 10 per cent. The three main antibiotics used to treat this disease are streptomycin, tetracycline and chloramphenicol. The first is still the drug of choice in several endemic countries, while the third is often restricted to meningeal forms. Administration of mono-, bi-, or tri-therapies depends on the clinical form of plague and on the severity of the symptoms. Today, these three antibiotics are no longer available in some countries and therefore two other molecules, gentamicin and fluoroquinolones, are used as alternatives to the former treatments. However, it should be kept in mind that these molecules have not yet been used to treat patients on a large scale.

Until recently, the plague bacillus was considered as universally susceptible to all antibiotics used to treat Gram-negative infections. In 1995, the first multi-resistant strain of Yersinia pestis was isolated in Madagascar from a patient with symptoms of bubonic plague. This resistance involved all the antibiotics recommended for plague therapy (streptomycin, tetracycline and chloramphenicol) and prophylaxis (sulphonamides and tetracycline), and also included antibiotics that may have represented therapeutic alternatives such as ampicillin, kanamycin, spectinomycin, and minocycline. The resistance determinants were carried by a conjugative plasmid which could be transferred to new Yersinia pestis strains at high frequencies. ${ }^{16}$ The acquisition of antibiotic resistance plasmids by Yersinia pestis was subsequently shown to occur in the flea intestine. ${ }^{17}$ Another point of significant public health concern was the recent discovery that the multi drug resistance plasmid is broadly disseminated among enterobacteria commonly isolated from food samples. ${ }^{18}$

\section{Conclusion}

The study of modern plague teaches us that, although this disease may take on numerous clinical forms, its diagnosis is still relatively easily made by local people in endemic areas

\footnotetext{
${ }^{16}$ M Galimand, A Guiyoule, G Gerbaud, B Rasoamanana, S Chanteau, E Carniel, and P Courvalin, 'Multidrug resistance in Yersinia pestis mediated by a transferable plasmid', N. Engl. J. Med., 1997, 337 (10): 677-80.

${ }^{17}$ B J Hinnebusch, M-L Rosso, T G Schwan, and E Carniel, 'High-frequency conjugative transfer of antibiotic resistance genes to Yersinia pestis in the flea midgut', Molecular Microbiology, 2002, 46 (2): 349-54.

${ }^{18} \mathrm{~T}$ Welch, W F Fricke, P F McDermott, D G White, M-L Rosso, et al., 'Multiple antimicrobial resistance in plague: an emerging public health risk', PLoS One, 2007, 2 (3): e309.
} 


\section{Elisabeth Carniel}

because the overall clinical presentation is recognizable and different from any other infection.

The comparison of the current plague foci in the world indicates that the type of flea vectors, rodent reservoirs, climates, ecological landscapes and seasonality varies considerably from one place to another. Since the characteristics of modern plague foci are so different, it is hardly possible to compare them with those of medieval plague. The climate, fleas and rodents in Europe during the Middle Ages were obviously different from those found in plague foci in Africa, Asia or America today. The ethnic group might also influence the susceptibility to plague, as observed for several other diseases, including AIDS. The human mode of living (chronic and abundant carriage of fleas, people living in close contact, often in cities, etc.) is also quite different from the situation met in the current endemic foci. Finally, the discovery of the aetiological agent, mode of transmission, rodent reservoir, and the advent of antibiotics during the twentieth century have considerably changed both the mode of human infection and the clinical presentations. Knowing that the plague is a flea-borne infection has allowed us to implement simple measures, such as disinsectization, thereby better controlling the spread of the disease at a local level. The availability of effective treatments has drastically reduced the frequency of the most severe forms of infections, thus changing the general clinical presentation.

Whether the devastating disease that killed a large portion of the human population during the Middle Ages was plague (i.e. a Yersinia pestis infection) is currently a matter of debate. Although we cannot prove anything one way or the other, it should be emphasized that it is not possible to reject the plague aetiology of the Black Death simply because certain symptoms and epidemiological features do not match those found today. 